\title{
Anti-windup compensation design for a class of distributed time-delayed cellular neural networks
}

\author{
HE Hanlin ${ }^{1}$, ZHA Miao ${ }^{1, *}$, and BIAN Shaofeng ${ }^{2}$ \\ 1. Department of Basic Courses, Naval University of Engineering, Wuhan 430033, China; \\ 2. Department of Navigation Engineering, Naval University of Engineering, Wuhan 430033, China
}

\begin{abstract}
Both time-delays and anti-windup (AW) problems are conventional problems in system design, which are scarcely discussed in cellular neural networks (CNNs). This paper discusses stabilization for a class of distributed time-delayed CNNs with input saturation. Based on the Lyapunov theory and the Schur complement principle, a bilinear matrix inequality (BMI) criterion is designed to stabilize the system with input saturation. By matrix congruent transformation, the BMI control criterion can be changed into linear matrix inequality (LMI) criterion, then it can be easily solved by the computer. It is a one-step AW strategy that the feedback compensator and the AW compensator can be determined simultaneously. The attraction domain and its optimization are also discussed. The structure of CNNs with both constant timedelays and distribute time-delays is more general. This method is simple and systematic, allowing dealing with a large class of such systems whose excitation satisfies the Lipschitz condition. The simulation results verify the effectiveness and feasibility of the proposed method.
\end{abstract}

Keywords: anti-windup (AW), cellular neural networks (CNNs), Lyapunov theory, linear matrix inequality (LMI), attraction domain.

DOI: $10.21629 / J S E E .2019 .06 .15$

\section{Introduction}

Artificial intelligence is a hot topic. With the development of science and technology, people hope to design machines which can simulate complex activities of the human brain. That comes the neural network [1,2]. The cellular neural network $(\mathrm{CNN})$ is one of the neural network models, which was first put forward by Chua and Yang [3]. It now has been successfully applied to many scientific fields [2,410]. CNNs are efficient tools for image analysis and pattern recognition [11]. Based on elementary cells connected to neighboring units, they are easy to install in hardware,

\footnotetext{
Manuscript received January 10, 2019.

*Corresponding author.

This work was supported by the National Natural Science Foundation of China (61374003; 41631072) and the Academic Foundation of Naval University of Engineering (20161475).
}

carrying out massively parallel processes. Another advantage of CNN is its ultra-fast precession on embedded platforms such as field programmable gate arrays (FPGAs) and graphic processing units (GPUs). Those merits make CNNs popular. Machot [9] applied this in real-time raindrop detection. Pierre [12] applied it in atmospheric dispersion modeling. In these applications, it is necessary to ensure the asymptotic stability of equilibrium points [13]. However, the oscillation phenomenon or network instability may be induced by time-delays in the interaction between the neurons when the neural networks are implemented by very large-scale integrated (VLSI) electronic circuits. Besides, owning to the physical and technological constraints, the saturation problem may occur when systems are designed with improper parameters. It also results in undesirable oscillatory behavior or instability.

Time-delays are inevitable in electronic implementation of CNNs. There are many excellent results on stability of CNNs with time-delays [2,14-19]. However, the time-delays mentioned in the above papers are constant time delays, proportional delays or time-varying delays. It is unreasonable that time delays are assumed to be discrete. Time-delays are continuously distributed. It is obvious that the distant past has less influence on the current state than the near past. Over a certain duration of time, the cumulative effect of time-delays distributed continuously cannot be ignored in high accuracy applications. To complement the study on time-delays, distributed time-delays have aroused peoples' attention [20-27]. Most of the papers [21 -26] are discussing global stability of CNNs with distributed time-delays. The condition is conservative in practical applications, so it still needs more study.

Saturation problems are common in practical application. Actuators which deliver the control signals are always subject to limits in magnitude or rate [28]. In CNNs, control signals may reach such limits when systems are designed with improper parameters. Then some people [29$31]$ began to focus on saturation problems in neural net- 
works. Actually, anti-windup (AW) research has a long history. Plenty of excellent work has been done [32-45]. Several methods now exist to handle saturation effects, but the most popular and effective one remains the AW approach [32]. The AW approach has been developed into two kinds: one-step method and two-step method. An overview of these results can be found in the survey [13]. Those two methods have their own merits and demerits [35-45]. Li et al. [41] proposed an AW based robust dynamic output feedback control algorithm for hydraulic servo position system with input saturation in a rolling mill. By using the Finsler's lemma, the controller parameter matrices are obtained by solving linear matrix inequalitys (LMIs) where the saturation is ignored. Then the saturation is considered by designing an AW-based robust dynamic output feedback controller, which uses the Lyapunov method to keep the stability of the system. It is a two-step AW method. The two-step AW method is more popular in history [41,43-45]. Designing the controller directly without considering saturation makes this method universal in practice. However, it has some drawbacks. First, only sub-optimal solution can be achieved since the controller and its AW compensator are designed separately. Second, the effect of the nominal controller on the closed-loop performance under saturation is completely ignored [42]. The one-step AW method [38,42] can overcome those drawbacks. Tahoun [38] proposed the control technique using both the state-feedback and the outputfeedback methodologies. It is a one-step AW method. The $\mathrm{AW}$ gain is derived along with system parameters, and the results are more optical than the two-step AW methods assuming AW gain offline.

Both time-delays and saturation can damage the stability of CNNs. However, there are few results on taking account of CNNs with distributed time-delays and saturation. We can find some works considering both two factors in other systems. Qi et al. [39] studied the problem of AW design for stochastic Markovian switching systems with mode-dependent time-varying delays and saturation nonlinearity. Both problems are discussed but not the CNNs system. He et al. [46] studied the problem of guaranteed cost AW stabilization of discrete delayed CNNs. It is a two-step AW method. Both time-delays and input saturation were mentioned but it did not discuss the distribute time-delays. Zha et al. [47] proposed an algebraic antiwindup compensation design for a class of time-delayed CNNs. It is similar to this paper but it did not discuss the distribute time-delays. Consequently, the stabilization for CNNs with distribute time-delays and input saturation still requires further consideration.

Motivated by the above-mentioned discussion, an AW design for a class of distributed time-delayed CNNs with input saturation is discussed in this paper. The structure of CNNs with both constant time-delays and distribute timedelays is more general. There are few results on AW research for this kind of systems. By employing the Lyapunov theory, the Schur complement principle and the LMI technique, an algebraic criterion is proposed for the design of the AW compensator. The attraction domain and its optimization are also presented. Specifically, the obtained method is an optimal solution for both feedback compensator and AW compensator parameters, which can be worked out simultaneously by solving certain LMIs. The results are new and they complement the previous study on CNNs.

This paper is organized as follows. In Section 2, system description and preliminaries are stated and some lemmas are listed. The problem to be treated is formally stated. In Section 3, the theoretical results allowing to determine the feedback gain, the AW gain and an associated region of stability are presented based on the Lyapunov stability theory. Section 4 talks about the method on transforming the stability condition into the LMI form and enlarging the basin of attraction of the closed-loop system. The effectiveness of the proposed approach is illustrated by examples in Section 5.

Notations. For a real symmetric matrix means $M, M>$ 0 and $\boldsymbol{M}<0$ respectively means the matrix is positive definite and negative definite. $\boldsymbol{M}^{\mathrm{T}}$ denotes the transpose of the matrix $\boldsymbol{M}$. The symbol $\boldsymbol{M}_{i}$ denotes the $i$ th row of matrix $\boldsymbol{M}$. Here, $\operatorname{He}(\boldsymbol{M})=\boldsymbol{M}+\boldsymbol{M}^{\mathrm{T}}$ for the real square matrix $M$, * stands for the symmetric blocks. $I$ denotes an $n$-dimensional identity matrix. $\operatorname{diag}(\boldsymbol{A}, \boldsymbol{B})$ is a blockdiagonal matrix.

\section{Model formulation and preliminaries}

Consider the following kind of distributed time-delayed CNNs:

$$
\begin{gathered}
\dot{\boldsymbol{x}}(t)=-\boldsymbol{A} \boldsymbol{x}(t)+\boldsymbol{M} f(\boldsymbol{x}(t))+\boldsymbol{B} f\left(\boldsymbol{x}\left(t-\tau_{1}(t)\right)\right)+ \\
\boldsymbol{C} \int_{t-\tau_{2}}^{t} f(\boldsymbol{x}(s)) \mathrm{d} s+\boldsymbol{G} \operatorname{sat}(\boldsymbol{u}(t))
\end{gathered}
$$

where $\boldsymbol{x}(t)=\left(x_{1}(t), x_{2}(t), \ldots, x_{n}(t)\right)^{\mathrm{T}}$ represents the state vector associated with the neurons; $n$ denotes the number of units in CNNs; $\boldsymbol{A}=\operatorname{diag}\left(a_{1}, a_{2}, \ldots, a_{n}\right)$ $\left(a_{i}>0, i=1,2, \ldots, n\right)$ represents the rate with which the neuron will reset its potential to the testing state in isolation when disconnected from the network and external inputs. $\boldsymbol{M}=\left(m_{i j}\right), \boldsymbol{B}=\left(b_{i j}\right), \boldsymbol{C}=\left(c_{i j}\right)$, which belong to $\mathbf{R}^{n \times n}$ are the interconnection matrices representing the weighting coefficients of the neurons, and $\boldsymbol{G}=\left(g_{i j}\right) \in$ $\mathbf{R}^{n \times m}$ represents the control gain matrix of the system. $f(\boldsymbol{x}(\cdot))=\left(f\left(x_{1}(\cdot)\right), f\left(x_{2}(\cdot)\right), \ldots, f\left(x_{n}(\cdot)\right)\right)^{\mathrm{T}}$ are the associated neuron activation functions, and $f\left(x_{i}(\cdot)\right)=$ 
$\left(\left|x_{i}(\cdot)+1\right|-\left|x_{i}(\cdot)-1\right|\right) / 2(i=1,2, \ldots, n) \cdot \tau_{1}(t) \geqslant 0$ corresponds to the transmission delay of neurons at the time $t$, whose time derivative satisfying $\dot{\tau}_{1}(t) \leqslant \eta<1$, and $\tau_{2}>0$ is constant. $\boldsymbol{u}(t)$ denotes the external inputs; $\operatorname{sat}(\boldsymbol{u}(t))=\left(\operatorname{sat}\left(u_{1}(t)\right), \operatorname{sat}\left(u_{2}(t)\right), \ldots, \operatorname{sat}\left(u_{m}(t)\right)\right)$ represents the vector valued saturation function, where $\operatorname{sat}\left(u_{i}\right)=\operatorname{sign}\left(u_{i}\right) \min \left\{u_{0 i},\left|u_{i}\right|\right\}, u_{0 i}>0(i=$ $1,2, \ldots, m)$.

Representing (1) by scalar equations, we can get

$$
\begin{gathered}
\dot{x}_{i}(t)=-a_{i} x_{i}(t)+\sum_{j=1}^{n} m_{i j} f\left(x_{j}(t)\right)+ \\
\sum_{j=1}^{n} b_{i j} f\left(x_{j}\left(t-\tau_{1}(t)\right)\right)+\sum_{j=1}^{n} c_{i j} \int_{t-\tau_{2}}^{t} f\left(x_{j}(s)\right) \mathrm{d} s+ \\
\sum_{j=1}^{m} g_{i j} \operatorname{sat}\left(u_{j}(t)\right), \quad i=1,2, \ldots, n .
\end{gathered}
$$

Let us define a dead zone nonlinear function $\psi(\boldsymbol{u})=$ $\psi(-\boldsymbol{K} \boldsymbol{x}(t))=-\boldsymbol{K} \boldsymbol{x}(t)-\operatorname{sat}(-\boldsymbol{K} \boldsymbol{x}(t))$, and then we have sat $(-\boldsymbol{K} \boldsymbol{x}(t))=-\boldsymbol{K} \boldsymbol{x}(t)-\psi(-\boldsymbol{K} \boldsymbol{x}(t))$. In order to mitigate the undesirable effects of windup caused by input saturation, we can add an AW compensator $\boldsymbol{E}_{c} \psi(-\boldsymbol{K} \boldsymbol{x}(t))$ to (1), so we have the following closedloop system:

$$
\begin{gathered}
\dot{\boldsymbol{x}}(t)=-\boldsymbol{A} \boldsymbol{x}(t)+\boldsymbol{M} f(\boldsymbol{x}(t))+\boldsymbol{B} f\left(\boldsymbol{x}\left(t-\tau_{1}(t)\right)\right)+ \\
\boldsymbol{C} \int_{t-\tau_{2}}^{t} f(\boldsymbol{x}(s)) \mathrm{d} s+\boldsymbol{G} \operatorname{sat}(-\boldsymbol{K} \boldsymbol{x}(t))+\boldsymbol{E}_{c} \psi(-\boldsymbol{K} \boldsymbol{x}(t))
\end{gathered}
$$

or

$$
\begin{gathered}
\dot{\boldsymbol{x}}(t)=-(\boldsymbol{G} \boldsymbol{K}+\boldsymbol{A}) \boldsymbol{x}(t)+\boldsymbol{M} f(\boldsymbol{x}(t))+ \\
\boldsymbol{B} f\left(\boldsymbol{x}\left(t-\tau_{1}(t)\right)\right)+\left(\boldsymbol{E}_{c}-\boldsymbol{G}\right) \psi(-\boldsymbol{K} \boldsymbol{x}(t))+ \\
\boldsymbol{C} \int_{t-\tau_{2}}^{t} f(\boldsymbol{x}(s)) \mathrm{d} s
\end{gathered}
$$

where $\boldsymbol{E}_{c}$ represents the gain matrix of AW. Let $\boldsymbol{N}_{i}$ and $\boldsymbol{K}_{i}$ represent the $i$ th rows of matrices $\boldsymbol{N}$ and $\boldsymbol{K}$, and we define the polyhedral set

$$
\boldsymbol{F}=\left\{\boldsymbol{x}(t)||\left(-\boldsymbol{K}_{i}-\boldsymbol{N}_{i} \boldsymbol{P}\right) \boldsymbol{x}(t) \mid \leqslant u_{i \max }\right\} .
$$

Our goal is to find out a proper AW compensator so that system (4) would be stable. We also appreciate a larger attraction domain. The problem we aim to solve throughout this note is then summarized as follows.

Problem 1 Determine the matrices $\boldsymbol{K}, \boldsymbol{E}_{c}$ and a region of asymptotic stability, as large as possible, for the closed-loop system (4).

Before proceeding, the following two lemmas will be introduced.

Lemma 1 [32] Let $\boldsymbol{P}$ be an $n \times n$ symmetric positive definite matrix, and let $\boldsymbol{T}$ be an $n \times n$ diagonal matrix. Then, the following matrix inequality holds:

$$
\psi^{\mathrm{T}}(-\boldsymbol{K} \boldsymbol{x}(t)) \boldsymbol{T}(\boldsymbol{N} \boldsymbol{P} \boldsymbol{x}(t)-\psi(-\boldsymbol{K} \boldsymbol{x}(t))) \geqslant 0
$$

Lemma 2 [48] Suppose that $\boldsymbol{X}_{22}<0$ and $\boldsymbol{D}>0$. Then the following quadratic matrix inequality

$$
\left[\begin{array}{cc}
\boldsymbol{X}_{11}+\boldsymbol{H}^{\mathrm{T}} \boldsymbol{D} \boldsymbol{H} & \boldsymbol{X}_{12} \\
* & \boldsymbol{X}_{22}
\end{array}\right]<0
$$

holds if and only if

$$
\left[\begin{array}{ccc}
\boldsymbol{X}_{11} & \boldsymbol{H}^{\mathrm{T}} & \boldsymbol{X}_{12} \\
* & -\boldsymbol{D}^{-1} & \mathbf{0} \\
* & * & \boldsymbol{X}_{22}
\end{array}\right]<0
$$

where $\boldsymbol{X}_{11} \in \mathbf{R}^{p \times p}, \boldsymbol{X}_{12} \in \mathbf{R}^{p \times q}, \boldsymbol{X}_{22} \in \mathbf{R}^{q \times q}, \boldsymbol{H} \in$ $\mathbf{R}^{d \times q}, \boldsymbol{D} \in \mathbf{R}^{d \times d}$, and $\boldsymbol{X}_{11}^{\mathrm{T}}=\boldsymbol{X}_{11}, \boldsymbol{X}_{22}^{\mathrm{T}}=\boldsymbol{X}_{22}, \boldsymbol{D}^{\mathrm{T}}=$ D.

\section{Stability conditions in the form of bilinear matrix inequalitys (BMIs)}

Combined with Lemma 1, we can have the following theorem.

Theorem 1 If there exist positive definite matrices $\boldsymbol{P}$, $\boldsymbol{Q}$ and $\boldsymbol{R}$, matrices $\boldsymbol{E}_{c}, \boldsymbol{N}$ and a diagonal positive-definite matrix $\boldsymbol{T}$, such that

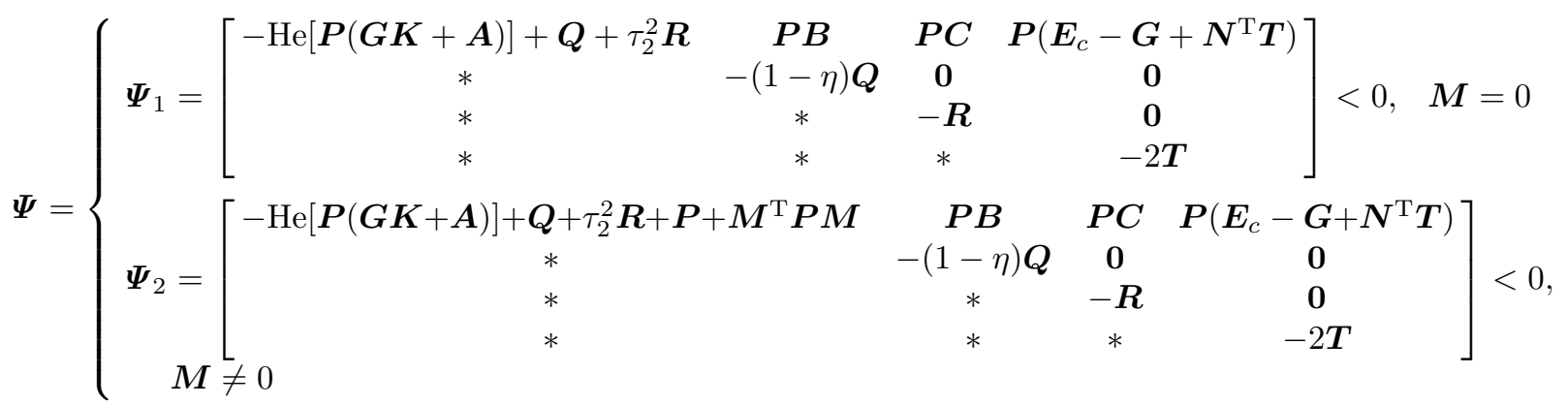


HE Hanlin et al.: Anti-windup compensation design for a class of distributed time-delayed cellular neural networks

$$
\left[\begin{array}{cc}
\boldsymbol{P} & \left(-\boldsymbol{K}_{i}^{\mathrm{T}}-\boldsymbol{P} \boldsymbol{N}_{i}^{\mathrm{T}}\right) \\
* & u_{0 i}^{2}
\end{array}\right] \geqslant 0, \quad i=1,2, \ldots, m \text {. }
$$

Then the ellipsoid $\boldsymbol{\Lambda}=\left\{\boldsymbol{x}(t) \mid \boldsymbol{x}^{\mathrm{T}}(t) \boldsymbol{P} \boldsymbol{x}(t) \leqslant 1\right\}$ is a domain of attraction for system (4).

Proof If (10) is satisfied, we have $u_{0 i}^{2}-\left(-\boldsymbol{K}_{i}-\right.$ $\left.\boldsymbol{N}_{i} \boldsymbol{P}\right) \boldsymbol{P}^{-1}\left(-\boldsymbol{K}_{i}^{\mathrm{T}}-\boldsymbol{P} \boldsymbol{N}_{i}^{\mathrm{T}}\right) \geqslant 0$ on account of the Schur's complement, which implies

$$
\frac{-\boldsymbol{K}_{i}-\boldsymbol{N}_{i} \boldsymbol{P}}{u_{0 i}} \boldsymbol{P}^{-1} \frac{-\boldsymbol{K}_{i}^{\mathrm{T}}-\boldsymbol{P} \boldsymbol{N}_{i}^{\mathrm{T}}}{u_{0 i}} \leqslant 1
$$

and we also have $\boldsymbol{x}^{\mathrm{T}}(t) \boldsymbol{P} \boldsymbol{x}(t) \leqslant 1$ for all $\boldsymbol{x}(t) \in \boldsymbol{\Lambda}$. Based on Holder inequality,

$$
\begin{gathered}
{\left[\frac{\left(-\boldsymbol{K}_{i}-\boldsymbol{N}_{i} \boldsymbol{P}\right)}{u_{0 i}} \boldsymbol{P}^{-\frac{1}{2}}\right] \cdot\left[\frac{\left(-\boldsymbol{K}_{i}-\boldsymbol{N}_{i} \boldsymbol{P}\right)}{u_{0 i}} \boldsymbol{P}^{-\frac{1}{2}}\right]^{\mathrm{T}} .} \\
\left(\boldsymbol{P}^{\frac{1}{2}} \boldsymbol{x}(t)\right)^{\mathrm{T}}\left(\boldsymbol{P}^{\frac{1}{2}} \boldsymbol{x}(t)\right)=\frac{\left(-\boldsymbol{K}_{i}-\boldsymbol{N}_{i} \boldsymbol{P}\right)}{u_{0 i}} \boldsymbol{P}^{-1} . \\
\frac{\left(-\boldsymbol{K}_{i}^{\mathrm{T}}-\boldsymbol{P} \boldsymbol{N}_{i}^{\mathrm{T}}\right)}{u_{0 i}} \cdot \boldsymbol{x}(t) \boldsymbol{P} \boldsymbol{x}(t) \leqslant 1,
\end{gathered}
$$

we can get $\left|\frac{\left(-\boldsymbol{K}_{i}-\boldsymbol{N}_{i} \boldsymbol{P}\right) \boldsymbol{x}(t)}{u_{0 i}}\right| \leqslant 1$, which implies the matrix inequality (6) holds.

Define a Lyapunov-Krasovskii functional candidate in the form of $V=V_{1}+V_{2}$, where

$\left|\frac{\left(-\boldsymbol{K}_{i}-\boldsymbol{N}_{i} \boldsymbol{P}\right) \boldsymbol{x}(t)}{u_{0 i}}\right|=\left|\frac{\left(-\boldsymbol{K}_{i}-\boldsymbol{N}_{i} \boldsymbol{P}\right)}{u_{0 i}} \boldsymbol{P}^{-\frac{1}{2}} \boldsymbol{P}^{\frac{1}{2}} \boldsymbol{x}(t)\right| \leqslant \quad V_{1}=\boldsymbol{x}^{\mathrm{T}}(t) \boldsymbol{P} \boldsymbol{x}(t)+\int_{t-\tau_{1}(t)}^{t} f(\boldsymbol{x}(s))^{\mathrm{T}} \boldsymbol{Q} f(\boldsymbol{x}(s)) \mathrm{d} s$

$$
V_{2}=\int_{t-\tau_{2}}^{t}\left\{\left[\int_{s}^{t} f(\boldsymbol{x}(\omega))^{\mathrm{T}} \mathrm{d} \omega\right] \boldsymbol{R}\left[\int_{s}^{t} f(\boldsymbol{x}(\omega)) \mathrm{d} \omega\right]\right\} \mathrm{d} s+\int_{0}^{\tau_{2}} \int_{t-s}^{t}(\omega-t+s) f(\boldsymbol{x}(\omega))^{\mathrm{T}} \boldsymbol{R} f(\boldsymbol{x}(\omega)) \mathrm{d} \omega \mathrm{d} s
$$

The time derivative of $V_{1}$ and $V_{2}$ evaluated along the solution of (4) is obtained as follows:

$$
\begin{gathered}
\dot{V}_{1} \leqslant 2 \boldsymbol{x}(t)^{\mathrm{T}} \boldsymbol{P}\left[-(\boldsymbol{G} \boldsymbol{K}+\boldsymbol{A}) \boldsymbol{x}(t)+\boldsymbol{M} f(\boldsymbol{x}(t))+\boldsymbol{B} f\left(\boldsymbol{x}\left(t-\tau_{1}(t)\right)\right)+\left(\boldsymbol{E}_{c}-\boldsymbol{G}\right) \psi(\boldsymbol{K} \boldsymbol{x}(t))+\right. \\
\left.\boldsymbol{C} \int_{t-\tau_{2}}^{t} f(\boldsymbol{x}(s)) \mathrm{d} s\right]+f(\boldsymbol{x}(t))^{\mathrm{T}} \boldsymbol{Q} f(\boldsymbol{x}(t))-(1-\eta) f\left(\boldsymbol{x}\left(t-\tau_{1}(t)\right)\right)^{\mathrm{T}} \boldsymbol{Q} f\left(\boldsymbol{x}\left(t-\tau_{1}(t)\right)\right)= \\
2 \boldsymbol{x}(t)^{\mathrm{T}} \boldsymbol{P}\left[-(\boldsymbol{G} \boldsymbol{K}+\boldsymbol{A}) \boldsymbol{x}(t)+\boldsymbol{B} f\left(\boldsymbol{x}\left(t-\tau_{1}(t)\right)\right)+\left(\boldsymbol{E}_{c}-\boldsymbol{G}\right) \psi(\boldsymbol{K} \boldsymbol{x}(t))+\boldsymbol{C} \int_{t-\tau_{2}}^{t} f(\boldsymbol{x}(s)) \mathrm{d} s\right]+ \\
2 \boldsymbol{x}(t)^{\mathrm{T}} \boldsymbol{P} \boldsymbol{M} f(\boldsymbol{x}(t))+f(\boldsymbol{x}(t))^{\mathrm{T}} \boldsymbol{Q} f(\boldsymbol{x}(t))-(1-\eta) f\left(\boldsymbol{x}\left(t-\tau_{1}(t)\right)\right)^{\mathrm{T}} \boldsymbol{Q} f\left(\boldsymbol{x}\left(t-\tau_{1}(t)\right)\right) .
\end{gathered}
$$

For $\boldsymbol{P}$ is the positive definite matrix and $\left|f\left(\boldsymbol{x}_{i}(t)\right)\right| \leqslant\left|\boldsymbol{x}_{i}(t)\right|$, we have $f(\boldsymbol{x}(t))^{\mathrm{T}} \boldsymbol{P} f(\boldsymbol{x}(t)) \leqslant \boldsymbol{x}(t)^{\mathrm{T}} \boldsymbol{P} \boldsymbol{x}(t)$. When $M=0$, we have

$$
\begin{gathered}
\dot{V}_{1} \leqslant 2 \boldsymbol{x}(t)^{\mathrm{T}} \boldsymbol{P}\left[-(\boldsymbol{G} \boldsymbol{K}+\boldsymbol{A}) \boldsymbol{x}(t)+\boldsymbol{B} f\left(\boldsymbol{x}\left(t-\tau_{1}(t)\right)\right)+\left(\boldsymbol{E}_{c}-\boldsymbol{G}\right) \psi(\boldsymbol{K} \boldsymbol{x}(t))+\boldsymbol{C} \int_{t-\tau_{2}}^{t} f(\boldsymbol{x}(s)) \mathrm{d} s\right]+ \\
2 \boldsymbol{x}(t)^{\mathrm{T}} \boldsymbol{P} \boldsymbol{M} f(\boldsymbol{x}(t))+f(\boldsymbol{x}(t))^{\mathrm{T}} \boldsymbol{Q} f(\boldsymbol{x}(t))-(1-\eta) f\left(\boldsymbol{x}\left(t-\tau_{1}(t)\right)\right)^{\mathrm{T}} \boldsymbol{Q} f\left(\boldsymbol{x}\left(t-\tau_{1}(t)\right)\right) \leqslant \\
2 \boldsymbol{x}(t)^{\mathrm{T}} \boldsymbol{P}\left[-(\boldsymbol{G} \boldsymbol{K}+\boldsymbol{A}) \boldsymbol{x}(t)+\boldsymbol{B} f\left(\boldsymbol{x}\left(t-\tau_{1}(t)\right)\right)+\left(\boldsymbol{E}_{c}-\boldsymbol{G}\right) \psi(\boldsymbol{K} \boldsymbol{x}(t))+\boldsymbol{C} \int_{t-\tau_{2}}^{t} f(\boldsymbol{x}(s)) \mathrm{d} s\right]+ \\
\boldsymbol{x}(t)^{\mathrm{T}} \boldsymbol{Q} \boldsymbol{x}(t)-(1-\eta) f\left(\boldsymbol{x}\left(t-\tau_{1}(t)\right)\right)^{\mathrm{T}} \boldsymbol{Q} f\left(\boldsymbol{x}\left(t-\tau_{1}(t)\right)\right)
\end{gathered}
$$

When $M \neq 0$, we get

$$
2 \boldsymbol{x}(t)^{\mathrm{T}} \boldsymbol{P} \boldsymbol{M} f(\boldsymbol{x}(t)) \leqslant \boldsymbol{x}(t)^{\mathrm{T}} \boldsymbol{P} \boldsymbol{x}(t)+f(\boldsymbol{x}(t))^{\mathrm{T}} \boldsymbol{M}^{\mathrm{T}} \boldsymbol{P} \boldsymbol{M} f(\boldsymbol{x}(t)) \leqslant \boldsymbol{x}(t)^{\mathrm{T}}\left(\boldsymbol{P}+\boldsymbol{M}^{\mathrm{T}} \boldsymbol{P} \boldsymbol{M}\right) \boldsymbol{x}(t) .
$$

Then we have

$$
\begin{gathered}
\dot{V}_{1} \leqslant 2 \boldsymbol{x}(t)^{\mathrm{T}} \boldsymbol{P}\left[-(\boldsymbol{G K}+\boldsymbol{A}) \boldsymbol{x}(t)+\boldsymbol{B} f\left(\boldsymbol{x}\left(t-\tau_{1}(t)\right)\right)+\left(\boldsymbol{E}_{c}-\boldsymbol{G}\right) \psi(\boldsymbol{K} \boldsymbol{x}(t))+\boldsymbol{C} \int_{t-\tau_{2}}^{t} f(\boldsymbol{x}(s)) \mathrm{d} s\right]+ \\
2 \boldsymbol{x}(t)^{\mathrm{T}} \boldsymbol{P} \boldsymbol{M} f(\boldsymbol{x}(t))+f(\boldsymbol{x}(t))^{\mathrm{T}} \boldsymbol{Q} f(\boldsymbol{x}(t))-(1-\eta) f\left(\boldsymbol{x}\left(t-\tau_{1}(t)\right)\right)^{\mathrm{T}} \boldsymbol{Q} f\left(\boldsymbol{x}\left(t-\tau_{1}(t)\right)\right) \leqslant
\end{gathered}
$$




$$
\begin{gathered}
2 \boldsymbol{x}(t)^{\mathrm{T}} \boldsymbol{P}\left[-(\boldsymbol{G} \boldsymbol{K}+\boldsymbol{A}) \boldsymbol{x}(t)+\boldsymbol{B} f\left(\boldsymbol{x}\left(t-\tau_{1}(t)\right)\right)+\left(\boldsymbol{E}_{c}-\boldsymbol{G}\right) \psi(\boldsymbol{K} \boldsymbol{x}(t))+\boldsymbol{C} \int_{t-\tau_{2}}^{t} f(\boldsymbol{x}(s)) \mathrm{d} s\right]+ \\
\boldsymbol{x}(t)^{\mathrm{T}}\left(\boldsymbol{P}+\boldsymbol{M}^{\mathrm{T}} \boldsymbol{P} \boldsymbol{M}+\boldsymbol{Q}\right) \boldsymbol{x}(t)-(1-\eta) f\left(\boldsymbol{x}\left(t-\tau_{1}(t)\right)\right)^{\mathrm{T}} \boldsymbol{Q} f\left(\boldsymbol{x}\left(t-\tau_{1}(t)\right)\right) . \\
\dot{V}_{2}=-\left[\int_{t-\tau_{2}}^{t} f(\boldsymbol{x}(\omega))^{\mathrm{T}} \mathrm{d} \omega\right] \boldsymbol{R}\left[\int_{t-\tau_{2}}^{t} f(\boldsymbol{x}(\omega)) \mathrm{d} \omega\right]+2 \int_{t-\tau_{2}}^{t}\left(\omega-t+\tau_{2}\right) f(\boldsymbol{x}(t))^{\mathrm{T}} \boldsymbol{R} f(\boldsymbol{x}(\omega)) \mathrm{d} \omega+ \\
\int_{0}^{\tau_{2}} s f(\boldsymbol{x}(t))^{\mathrm{T}} \boldsymbol{R} f(\boldsymbol{x}(t)) \mathrm{d} s-\int_{0}^{\tau_{2}} \int_{t-s}^{t} f(\boldsymbol{x}(\omega))^{\mathrm{T}} \boldsymbol{R} f(\boldsymbol{x}(\omega)) \mathrm{d} \omega \mathrm{d} s .
\end{gathered}
$$

Combined with $2 \boldsymbol{X}^{\mathrm{T}} \boldsymbol{P} \boldsymbol{Y} \leqslant \boldsymbol{X}^{\mathrm{T}} \boldsymbol{P} \boldsymbol{X}+\boldsymbol{Y}^{\mathrm{T}} \boldsymbol{P} \boldsymbol{Y}$, we have

$$
\begin{gathered}
\dot{V}_{2} \leqslant-\left[\int_{t-\tau_{2}}^{t} f(\boldsymbol{x}(\omega))^{\mathrm{T}} \mathrm{d} \omega\right] \boldsymbol{R}\left[\int_{t-\tau_{2}}^{t} f(\boldsymbol{x}(\omega)) \mathrm{d} \omega\right]+\int_{t-\tau_{2}}^{t}\left(\omega-t+\tau_{2}\right)\left[f(\boldsymbol{x}(t))^{\mathrm{T}} \boldsymbol{R} f(\boldsymbol{x}(t))+f(\boldsymbol{x}(\omega))^{\mathrm{T}} \boldsymbol{R} f(\boldsymbol{x}(\omega))\right] \mathrm{d} \omega+ \\
\frac{1}{2} \tau_{2}^{2} f(\boldsymbol{x}(t))^{\mathrm{T}} \boldsymbol{R} f(\boldsymbol{x}(t))-\int_{t-\tau_{2}}^{t} \mathrm{~d} \omega \int_{t-\omega}^{\tau_{2}} f(\boldsymbol{x}(\omega))^{\mathrm{T}} \boldsymbol{R} f(\boldsymbol{x}(\omega)) \mathrm{d} s \leqslant-\left[\int_{t-\tau_{2}}^{t} f(\boldsymbol{x}(\omega))^{\mathrm{T}} \mathrm{d} \omega\right] \boldsymbol{R}\left[\int_{t-\tau_{2}}^{t} f(\boldsymbol{x}(\omega)) \mathrm{d} \omega\right]+ \\
\int_{t-\tau_{2}}^{t}\left(\omega-t+\tau_{2}\right)\left[f(\boldsymbol{x}(\omega))^{\mathrm{T}} \boldsymbol{R} f(\boldsymbol{x}(\omega))\right] \mathrm{d} \omega+\frac{1}{2} \tau_{2}^{2} f(\boldsymbol{x}(t))^{\mathrm{T}} \boldsymbol{R} f(\boldsymbol{x}(t))+\frac{1}{2} \tau_{2}^{2} f(\boldsymbol{x}(t))^{\mathrm{T}} \boldsymbol{R} f(\boldsymbol{x}(t))- \\
\int_{t-\tau_{2}}^{t}\left(\tau_{2}+\omega-t\right)\left[f(\boldsymbol{x}(\omega))^{\mathrm{T}} \boldsymbol{R} f(\boldsymbol{x}(\omega))\right] \mathrm{d} \omega \leqslant \tau_{2}^{2} f(\boldsymbol{x}(t))^{\mathrm{T}} \boldsymbol{R} f(\boldsymbol{x}(t))-\left[\int_{t-\tau_{2}}^{t} f(\boldsymbol{x}(\omega))^{\mathrm{T}} \mathrm{d} \omega\right] \boldsymbol{R}\left[\int_{t-\tau_{2}}^{t} f(\boldsymbol{x}(\omega)) \mathrm{d} \omega\right] \leqslant \\
\tau_{2}^{2} \boldsymbol{x}(t)^{\mathrm{T}} \boldsymbol{R} \boldsymbol{x}(t)-\left[\int_{t-\tau_{2}}^{t} f(\boldsymbol{x}(\omega))^{\mathrm{T}} \mathrm{d} \omega\right] \boldsymbol{R}\left[\int_{t-\tau_{2}}^{t} f(\boldsymbol{x}(\omega)) \mathrm{d} \omega\right] .
\end{gathered}
$$

By using (6), the time derivative of $V$ evaluated along the solution of (4) is obtained as

$$
\dot{V}=\dot{V}_{1}+\dot{V}_{2} \leqslant \boldsymbol{\xi}^{\mathrm{T}} \boldsymbol{\Psi} \boldsymbol{\xi}
$$

where $\boldsymbol{\xi}=\left[\boldsymbol{x}(t)^{\mathrm{T}} f\left(\boldsymbol{x}\left(t-\tau_{1}(t)\right)\right)^{\mathrm{T}} \int_{t-\tau_{2}}^{t} f(\boldsymbol{x}(s))^{\mathrm{T}} \mathrm{d} s\right.$ $\left.\psi(-\boldsymbol{K} \boldsymbol{x}(t))^{\mathrm{T}}\right]^{\mathrm{T}}$.

Hence, if (9) is satisfied, we have $\dot{V}<0$.
Thus, it is obvious that the ellipsoid $\boldsymbol{\Lambda}=$ $\left\{\boldsymbol{x}(t) \mid \boldsymbol{x}^{\mathrm{T}}(t) \boldsymbol{P} \boldsymbol{x}(t) \leqslant 1\right\}$ is the domain of attraction for system (4). This completes the proof of Theorem 1 .

In Theorem 1, if $-\boldsymbol{K}_{i}-\boldsymbol{N}_{i} \boldsymbol{P}=0$, for any $\boldsymbol{x}(t) \in \mathbf{R}^{n}$, Lemma 1 will be satisfied. Hence, (10) can be left out. We can obtain the following corollary.

Corollary 1 If there exist positive definite matrices $\boldsymbol{P}, \boldsymbol{Q}, \boldsymbol{R}$, matrices $\boldsymbol{E}_{c}, \boldsymbol{K}$ and a diagonal positive-definite matrix $\boldsymbol{T}$, such that

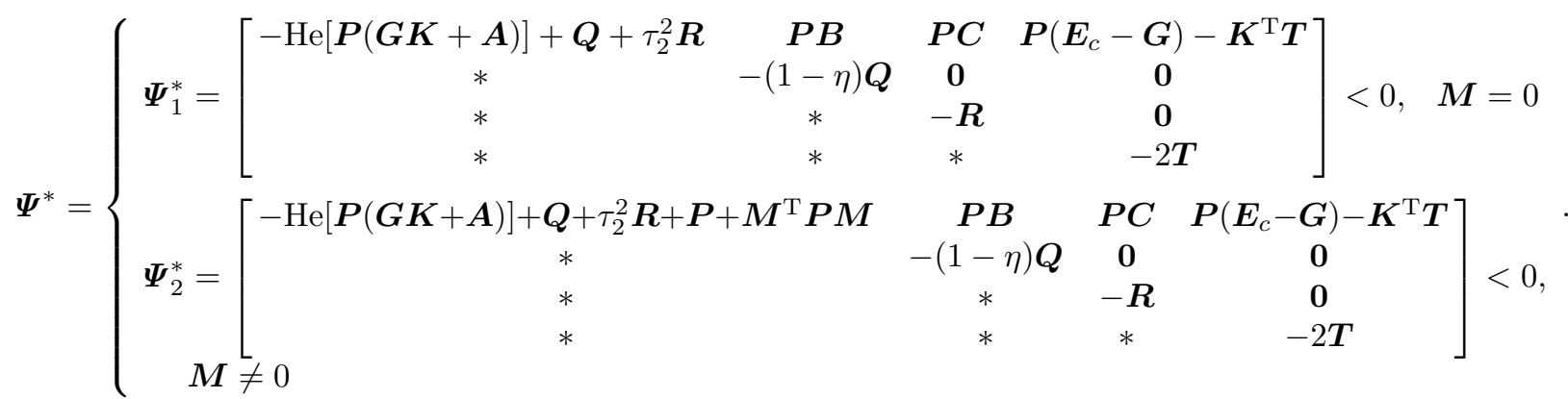

System (4) is globally asymptotic stable.

Remark 1 In the first row of the first column of the block matrix (20), it appears $\boldsymbol{Q}+\tau_{2}^{2} \boldsymbol{R}$. If we use the twostep AW method, though we can design the feedback con- trol gain matrix $\boldsymbol{K}$ to ensure $\boldsymbol{G} \boldsymbol{K}+\boldsymbol{A}$ to be Hurwitz matrix, we cannot ensure $-\mathrm{He}[\boldsymbol{P}(\boldsymbol{G} \boldsymbol{K}+\boldsymbol{A})]+\boldsymbol{Q}+\tau_{2}^{2} \boldsymbol{R}<0$. Therefore, the two-step AW method can not promise system (4) to be asymptotically stable. 
Remark $2 \quad \boldsymbol{P}$ is a positive definite matrix and $\left|f\left(\boldsymbol{x}_{i}(t)\right)\right| \leqslant\left|\boldsymbol{x}_{i}(t)\right|$. In designing $\dot{V}_{1}$ and $\dot{V}_{2}$, we apply the inequality $f(\boldsymbol{x}(t))^{\mathrm{T}} \boldsymbol{P} f(\boldsymbol{x}(t)) \leqslant \boldsymbol{x}(t)^{\mathrm{T}} \boldsymbol{P} \boldsymbol{x}(t)$, if $f(\boldsymbol{x}(t))$ is not a standard saturation function but a function with the Lipschitz constant $\lambda$ satisfying the Lipschitz condition. We can multiply the inequality by $\lambda^{2}$, the rest are the same as Theorem 1, so this theorem is established for a large class of such systems whose excitation satisfies the Lipschitz condition.

Remark 3 Using this kind of one-step AW strategy, $\boldsymbol{K}$ and $\boldsymbol{E}_{c}$ are worked out directly. The answer is the optimal solution.
Remark 4 For $\boldsymbol{M}=0,-\mathrm{He}[\boldsymbol{P}(\boldsymbol{G} \boldsymbol{K}+\boldsymbol{A})]+\boldsymbol{Q}+$ $\tau_{2}^{2} \boldsymbol{R} \neq-\mathrm{He}[\boldsymbol{P}(\boldsymbol{G K}+\boldsymbol{A})]+\boldsymbol{Q}+\tau_{2}^{2} \boldsymbol{R}+\boldsymbol{P}+\boldsymbol{M} \boldsymbol{P} \boldsymbol{M}$ so the AW strategy for distributed time-delayed CNNs (1) with $M=0$ has to be designed separately.

\section{Stability conditions in the form of LMIs}

Equations (9), (10) and (20) are bi-linear matrix inequalities which are not convenient to be solved. We will convert it into an LMI form to make it easily treated with the LMI software.

Theorem 2 There exist positive definite matrices $\boldsymbol{P}_{1}$, $Q_{1}, R_{1}$, a diagonal positive-definite matrix $\boldsymbol{T}_{1}$ and matrices $\boldsymbol{L}, \boldsymbol{J}, \boldsymbol{N}$, such that

$$
\boldsymbol{\Pi}=\left\{\begin{array}{c}
\boldsymbol{\Pi}_{1}=\left[\begin{array}{ccccc}
-\mathrm{He}\left[\left(\boldsymbol{G L}+\boldsymbol{A} \boldsymbol{P}_{1}\right)\right]+\boldsymbol{Q}_{1}+\tau_{2}^{2} \boldsymbol{R}_{1} & \boldsymbol{B} \boldsymbol{P}_{1} & \boldsymbol{C} \boldsymbol{P}_{1} & \boldsymbol{J}-\boldsymbol{G} \boldsymbol{T}_{1}+\boldsymbol{N}^{\mathrm{T}} \\
* & -(1-\eta) \boldsymbol{Q}_{1} & \mathbf{0} & \mathbf{0} \\
* & * & -\boldsymbol{R}_{1} & \mathbf{0} \\
* & * & * & -2 \boldsymbol{T}_{1}
\end{array}\right]<0, \quad \boldsymbol{M}=0 \\
\boldsymbol{\Pi}_{2}=\left[\begin{array}{ccccc}
-\mathrm{He}\left[\left(\boldsymbol{G L}+\boldsymbol{A} \boldsymbol{P}_{1}\right)\right]+\boldsymbol{Q}_{1}+\tau_{2}^{2} \boldsymbol{R}_{1}+\boldsymbol{P}_{1} & \boldsymbol{P}_{1} \boldsymbol{M}^{\mathrm{T}} & \boldsymbol{B} \boldsymbol{P}_{1} & \boldsymbol{C} \boldsymbol{P}_{1} & \left(\boldsymbol{J}-\boldsymbol{G} \boldsymbol{T}_{1}+\boldsymbol{N}^{\mathrm{T}}\right) \\
* & -\boldsymbol{P}_{1} & \mathbf{0} & \mathbf{0} & \mathbf{0} \\
* & * & -(1-\eta) \boldsymbol{Q}_{1} & \mathbf{0} & \mathbf{0} \\
* & * & * & -\boldsymbol{R}_{1} & \mathbf{0} \\
* & * & * & * & -2 \boldsymbol{T}_{1}
\end{array}\right]<0,
\end{array}\right.
$$

$$
\left[\begin{array}{cc}
\boldsymbol{P}_{1} & \left(-\boldsymbol{L}_{i}^{\mathrm{T}}-\boldsymbol{N}_{i}^{\mathrm{T}}\right) \\
* & u_{0 i}^{2}
\end{array}\right] \geqslant 0, \quad i=1,2, \ldots, m .
$$

If and only if there exist a positive definite matrix $\boldsymbol{P}, \boldsymbol{Q}, \boldsymbol{R}$, matrices $\boldsymbol{E}_{c}, \boldsymbol{N}$ and a diagonal positive-definite matrix $\boldsymbol{T}$, such that (9) and (10) are satisfied with $\boldsymbol{P}=$ $\boldsymbol{P}_{1}^{-1}, \boldsymbol{T}=\boldsymbol{T}_{1}^{-1}, \boldsymbol{Q}=\boldsymbol{P}_{1}^{-1} \boldsymbol{Q}_{1} \boldsymbol{P}_{1}^{-1}, \boldsymbol{R}=\boldsymbol{P}_{1}^{-1} \boldsymbol{R}_{1} \boldsymbol{P}_{1}^{-1}$,

$$
\left[\begin{array}{cc}
-\mathrm{He}[\boldsymbol{P}(\boldsymbol{G} \boldsymbol{K}+\boldsymbol{A})]+\boldsymbol{Q}+\tau_{2}^{2} \boldsymbol{R}+\boldsymbol{P} & \boldsymbol{M}^{\mathrm{T}} \\
* & -\boldsymbol{P}^{-1} \\
* & * \\
* & * \\
* & *
\end{array}\right.
$$

Pre- and post- multiplying (23) by $\operatorname{diag}\left(\boldsymbol{P}^{-1}, \boldsymbol{I}, \boldsymbol{P}^{-1}\right.$, $\left.\boldsymbol{P}^{-1}, \boldsymbol{T}^{-1}\right)$, we get $\boldsymbol{\Pi}_{2}<0$. Then we have (21). By the same reasoning, pre- and post-multiplying (10) by $\operatorname{diag}\left(\boldsymbol{P}^{-1}, 1\right)$, we have (22).

Necessity: if (21) and (22) holds true, by matrix congruent transformation, it is also easy to derive (9) and (10). It the feedback control gain matrix $\boldsymbol{K}=\boldsymbol{L} \boldsymbol{P}_{1}^{-1}$, and the AW control gain matrix $\boldsymbol{E}_{c}=\boldsymbol{J} \boldsymbol{T}_{1}^{-1}$.

Proof Sufficiency: if (9) holds, $\boldsymbol{M}=0$, pre- and postmultiplying $\boldsymbol{\Psi}_{1}$ by $\operatorname{diag}\left(\boldsymbol{P}^{-1}, \boldsymbol{P}^{-1}, \boldsymbol{P}^{-1}, \boldsymbol{T}^{-1}\right)$, we get $\boldsymbol{\Pi}_{1}<0 ; \boldsymbol{M} \neq 0$, combined with Lemma 2, $\boldsymbol{\Psi}_{2}$ in (9) is equivalent to (23):

$$
\left.\begin{array}{ccc}
\boldsymbol{P} \boldsymbol{B} & \boldsymbol{P} \boldsymbol{C} & \boldsymbol{P}\left(\boldsymbol{E}_{c}-\boldsymbol{G}+\boldsymbol{N}^{\mathrm{T}} \boldsymbol{T}\right) \\
0 & 0 & 0 \\
-(1-\eta) \boldsymbol{Q} & 0 & 0 \\
* & -\boldsymbol{R} & 0 \\
* & * & -2 \boldsymbol{T}
\end{array}\right]<0 .
$$

completes the proof.

Using the same method, Corollary 1 can be easily changed into LMI form.

Corollary 2 There exist positive definite matrices $\boldsymbol{P}_{1}$, $Q_{1}, R_{1}$, a diagonal positive-definite matrix $T_{1}$ and matrices $J, N$. 


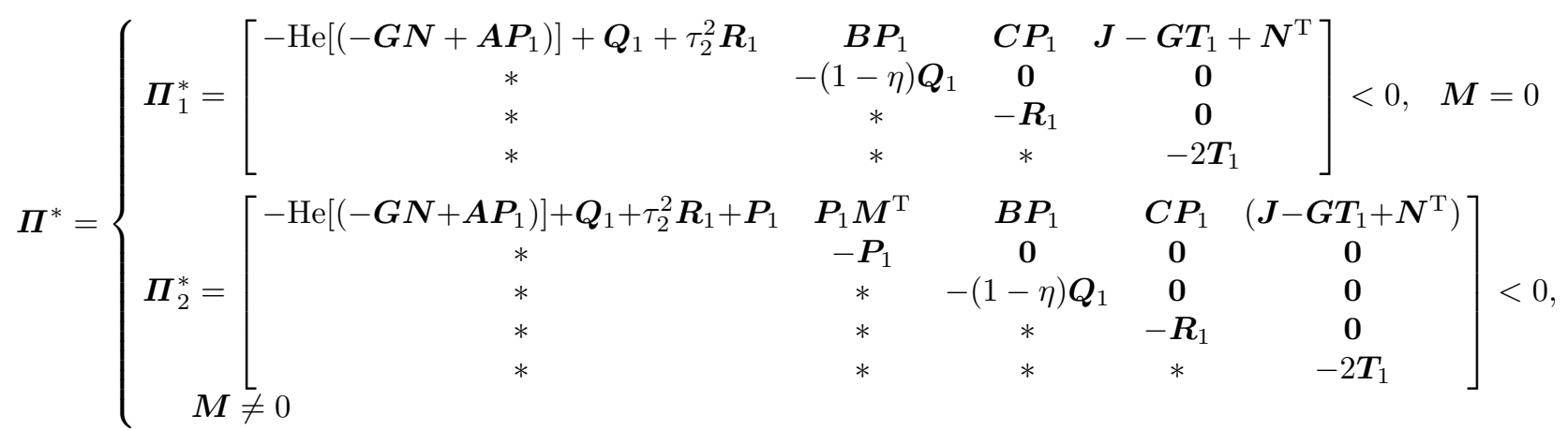

If and only if there exist a positive definite matrix $\boldsymbol{P}$, matrices $\boldsymbol{E}_{c}, \boldsymbol{K}$ and a diagonal positive-definite matrix $\boldsymbol{T}$, such that (9) is satisfied with $\boldsymbol{P}=\boldsymbol{P}_{1}^{-1}, \boldsymbol{T}=\boldsymbol{T}_{1}^{-1}$, $\boldsymbol{Q}=\boldsymbol{P}_{1}^{-1} \boldsymbol{Q}_{1} \boldsymbol{P}_{1}^{-1}, \boldsymbol{R}=\boldsymbol{P}_{1}^{-1} \boldsymbol{R}_{1} \boldsymbol{P}_{1}^{-1}$, the feedback control gain matrix $K=-N \boldsymbol{P}_{1}^{-1}$, and the AW control gain matrix $\boldsymbol{E}_{c}=\boldsymbol{J} \boldsymbol{T}_{1}^{-1}$.

Further, we want to compute the matrix $\boldsymbol{E}_{c}$ such that the associated region of asymptotic stability is as large as possible considering some size criterion. We take the method used in [32]. We should first give a set of admissible initial conditions $\boldsymbol{\Xi}_{0} \subset \mathbf{R}^{n}$, which can ensure systems to be asymptotically stable. Let $\Xi_{0}$ be defined as a polyhedral set described by the convex hull of its vertices

$$
\boldsymbol{\Xi}_{0} \triangleq C o\left\{\boldsymbol{v}_{1}, \boldsymbol{v}_{2}, \ldots, \boldsymbol{v}_{n_{r}}\right\}, \quad \boldsymbol{v}_{r} \in \mathbf{R}^{n} ; r=1, \ldots, n_{r} .
$$

Recalling Theorem 1, we aim at searching for $P, Q, R$, $\boldsymbol{E}_{c}, \boldsymbol{N}, \boldsymbol{T}$ in order to obtain $\beta \Xi_{0} \subset \Lambda(\boldsymbol{P}) . \beta$ is a scaling factor. $\Xi_{0}$ defines the directions in which we want to maximize $\Lambda$. Take $\beta=1$, and this problem reduces to a feasibility problem. In order to maximize the estimate of the basin of attraction associated to it, the goal will be to maximize $\beta$. Considering $\beta=1 / \sqrt{\mu}$, the minimization of $\mu$ implies the maximization of $\beta$. By solving the following convex optimization problem we can get a larger basin of attraction:

$$
\left\{\begin{array}{l}
\min _{\boldsymbol{P}_{1}, \boldsymbol{Q}_{1}, \boldsymbol{R}_{1}, \boldsymbol{L}, \boldsymbol{J}, N, \mu}^{\mu} \\
\text { s.t. }\left[\begin{array}{cc}
\mu & \boldsymbol{v}_{r}^{\mathrm{T}} \\
\boldsymbol{v}_{r} & \boldsymbol{P}_{1}
\end{array}\right] \geqslant 0, \quad r=1, \ldots, n_{r} . \\
\text { LMIs }(21) \text { and }(22)
\end{array}\right.
$$

\section{Illustrative examples}

In this section, examples are given to verify the effectiveness of the proposed methods.

Example 1 Distributed time-delayed CNN system with $M=0$

Consider the following distributed time-delayed CNN:

$$
\dot{\boldsymbol{x}}(t)=-\boldsymbol{A} \boldsymbol{x}(t)+\boldsymbol{B} f\left(\boldsymbol{x}\left(t-\tau_{1}(t)\right)\right)+
$$

$$
\boldsymbol{C} \int_{t-\tau_{2}}^{t} f(\boldsymbol{x}(s)) \mathrm{d} s, \quad i=1,2
$$

where $\boldsymbol{A}=\left[\begin{array}{ll}1 & \\ & 1\end{array}\right], \boldsymbol{B}=\left[\begin{array}{cc}1.8 & 0.1 \\ 25 & 1.92\end{array}\right], \boldsymbol{C}=$ $\left[\begin{array}{cc}-1.39 & 0.1 \\ 0.2 & -1.73\end{array}\right], \tau_{1}(t)=0.5 t, \tau_{2}=1, f(x)=$ $(|x+1|-|x-1|) / 2, f(\boldsymbol{x}(t))=\left(f\left(x_{1}(t)\right), f\left(x_{2}(t)\right)\right)^{\mathrm{T}}$, $f\left(\boldsymbol{x}\left(t-\tau_{1}(t)\right)\right)=\left(f\left(x_{1}\left(t-\tau_{1}(t)\right)\right), f\left(x_{2}\left(t-\tau_{1}(t)\right)\right)\right)^{\mathrm{T}}$, $u_{0 i}=1$. Supposing the initial states $\boldsymbol{x}_{0}=[-0.2,1]^{\mathrm{T}}$, the state trajectory of system (26) is shown in Fig. 1. When the input is saturation, choose $\boldsymbol{G}=\operatorname{diag}(1,1)$, an AW compensator $\boldsymbol{E}_{c} \psi(\boldsymbol{K} \boldsymbol{x}(t))$ is added to system (26), so we can get system (4). Combined with (21), (22) and correlative data, the feasible results with the help of the LMI toolbox of Matlab are obtained as follows:

$$
\begin{aligned}
\boldsymbol{P} & =\left[\begin{array}{ll}
8.7433 & 0.6253 \\
0.6253 & 0.5817
\end{array}\right], \\
\boldsymbol{Q} & =\left[\begin{array}{cc}
461.2609 & 35.0818 \\
35.0818 & 4.1258
\end{array}\right], \\
\boldsymbol{R} & =\left[\begin{array}{cc}
301.2754 & 22.8200 \\
22.8200 & 2.8482
\end{array}\right], \\
\boldsymbol{T} & =\left[\begin{array}{cc}
0.3935 & 0 \\
0 & 0.3935
\end{array}\right], \\
\boldsymbol{L} & =\left[\begin{array}{cc}
6.8197 & 2.3572 \\
-2.2020 & 6.9396
\end{array}\right], \\
\boldsymbol{J} & =\left[\begin{array}{cc}
9.1606 & -2.1595 \\
2.3997 & 8.7251
\end{array}\right], \\
\boldsymbol{N} & =\left[\begin{array}{cc}
-6.6192 & -2.3997 \\
2.1595 & -6.1838
\end{array}\right], \\
\boldsymbol{K} & =\left[\begin{array}{cc}
61.1012 & 5.6358 \\
-14.9137 & 2.6599
\end{array}\right], \\
\boldsymbol{E}_{c} & =\left[\begin{array}{cc}
3.6046 & -0.8498 \\
0.9443 & 3.4333
\end{array}\right] .
\end{aligned}
$$




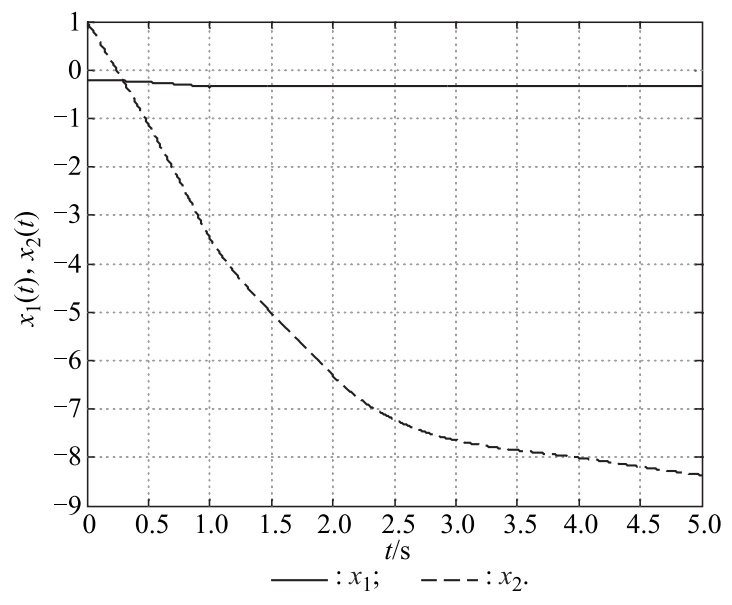

Fig. 1 Initial state trajectory of system (26) in Example 1

The states of $x_{1}$ and $x_{2}$ of system (4) are shown in Fig. 2. The control locus diagrams of system (4) are shown in Fig. 3.

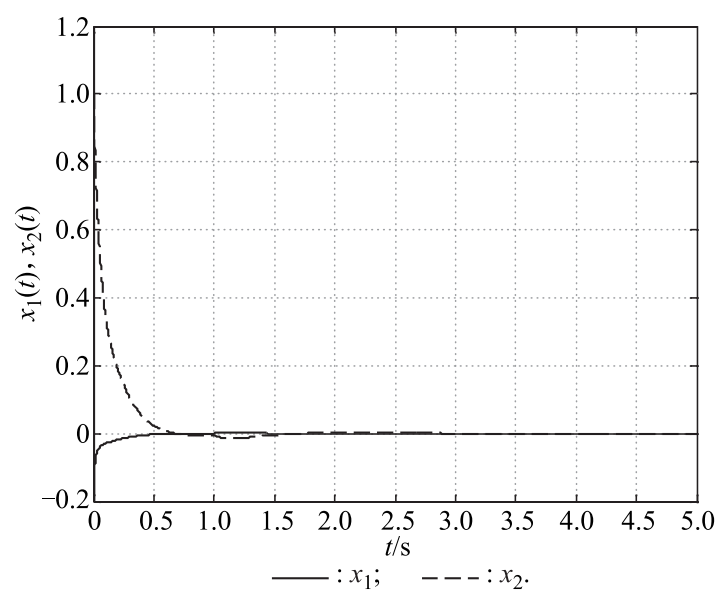

Fig. 2 State locus of system (4) with AW structure in Example 1

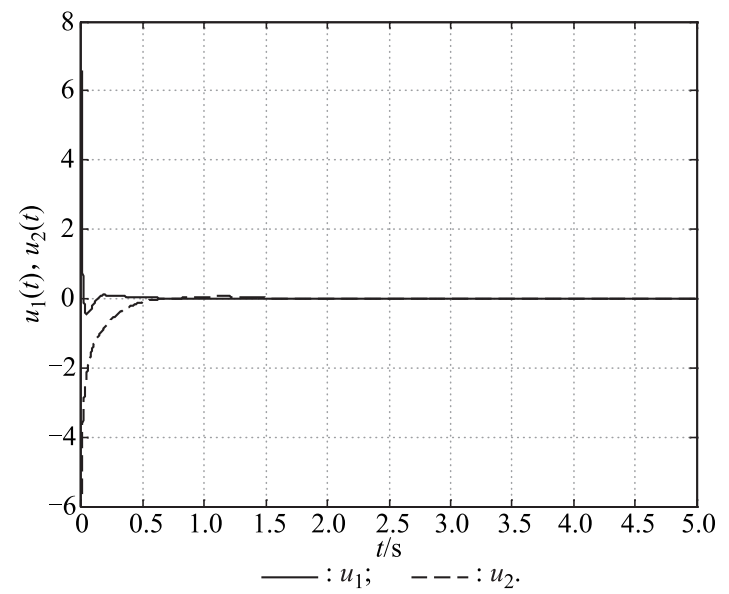

Fig. 3 Control locus of system (4) in Example 1

Comparing Fig. 1 and Fig. 2, the open-loop system (26) is unstable at the setting initial state. By adding the designed AW structure, the closed-loop system (4) becomes stable in a short time. The control locus of system (4) in Fig. 3 shows that $u_{1}(t)$ and $u_{2}(t)$ are big at the beginning. It is effective to make the state back to zero as soon as possible.

Let the shape set $\boldsymbol{\Xi}_{0}$ be defined by a square region in the space $\mathbf{R}^{2}$ :

$$
\boldsymbol{\Xi}_{0}=\operatorname{Co}\left\{\left[\begin{array}{l}
1 \\
1
\end{array}\right] ;\left[\begin{array}{c}
-1 \\
1
\end{array}\right] ;\left[\begin{array}{l}
-1 \\
-1
\end{array}\right] ;\left[\begin{array}{c}
1 \\
-1
\end{array}\right]\right\} \text {. }
$$

By solving (25), we can obtain another group of solution as follows:

$$
\begin{aligned}
& \boldsymbol{P}=\left[\begin{array}{ll}
0.0479 & 0.0030 \\
0.0030 & 0.0059
\end{array}\right] \\
& \boldsymbol{Q}=\left[\begin{array}{ll}
1.8378 & 0.1355 \\
0.1355 & 0.0214
\end{array}\right] \text {, } \\
& \boldsymbol{R}=\left[\begin{array}{ll}
0.6070 & 0.0418 \\
0.0418 & 0.0154
\end{array}\right] \\
& \boldsymbol{T}=\left[\begin{array}{cc}
0.0057 & 0 \\
0 & 0.0057
\end{array}\right] \\
& \boldsymbol{L}=\left[\begin{array}{cc}
644.4067 & 46.0232 \\
38.0651 & 787.5938
\end{array}\right] \\
& \boldsymbol{J}=\left[\begin{array}{cc}
816.5708 & 38.4129 \\
46.3878 & 954.7200
\end{array}\right] \text {, } \\
& N=\left[\begin{array}{cc}
-641.8539 & -46.3817 \\
-38.4129 & -780.0031
\end{array}\right] \text {, } \\
& \boldsymbol{K}=\left[\begin{array}{cc}
30.9732 & 2.2049 \\
4.1837 & 4.7702
\end{array}\right] \\
& \boldsymbol{E}_{c}=\left[\begin{array}{ll}
4.6737 & 0.2199 \\
0.2655 & 5.4644
\end{array}\right], \quad \mu=0.0948 .
\end{aligned}
$$

The estimation of the domain of attraction of system (4) is shown in Fig. 4. In Fig. 4, one locus is the estimation of the domain of attraction before optimization (discrete), the other is the estimation of the domain of attraction after optimization (continuous).

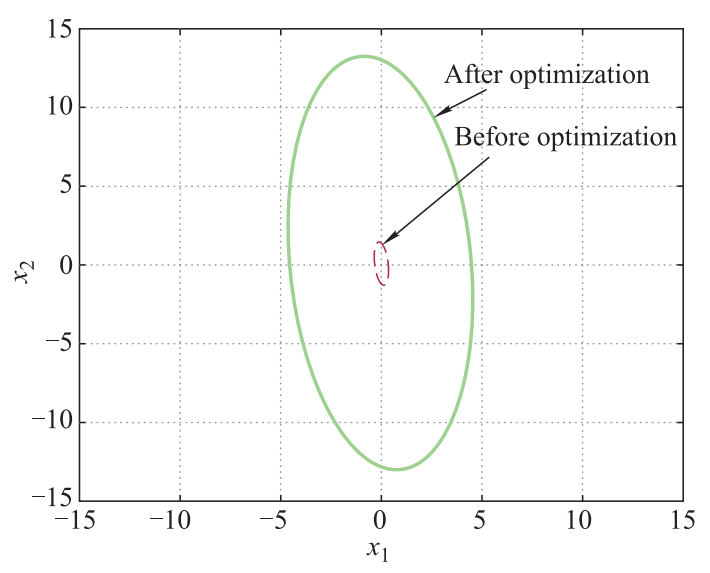

Fig. 4 Domain of attraction in Example 1 
It is obvious that by solving (25), we obtain a larger estimate of the domain of attraction of (4).

Example 2 Distributed time-delayed CNN system with $M \neq 0$

$$
\begin{gathered}
\dot{\boldsymbol{x}}(t)=-\boldsymbol{A} \boldsymbol{x}(t)+\boldsymbol{M} f(\boldsymbol{x}(t))+\boldsymbol{B} f\left(\boldsymbol{x}\left(t-\tau_{1}(t)\right)\right)+ \\
\boldsymbol{C} \int_{t-\tau_{2}}^{t} f(\boldsymbol{x}(s)) \mathrm{d} s, \quad i=1,2
\end{gathered}
$$

where $\boldsymbol{M}=\left[\begin{array}{cc}2.1 & -1.6 \\ 2.5 & 0.2\end{array}\right]$, and the rest settings are the same as Example 1. Suppose the initial states $\boldsymbol{x}_{0}=$ $[-0.2,0.9]^{\mathrm{T}}$, the state trajectory of system (27) is shown in Fig. 5.

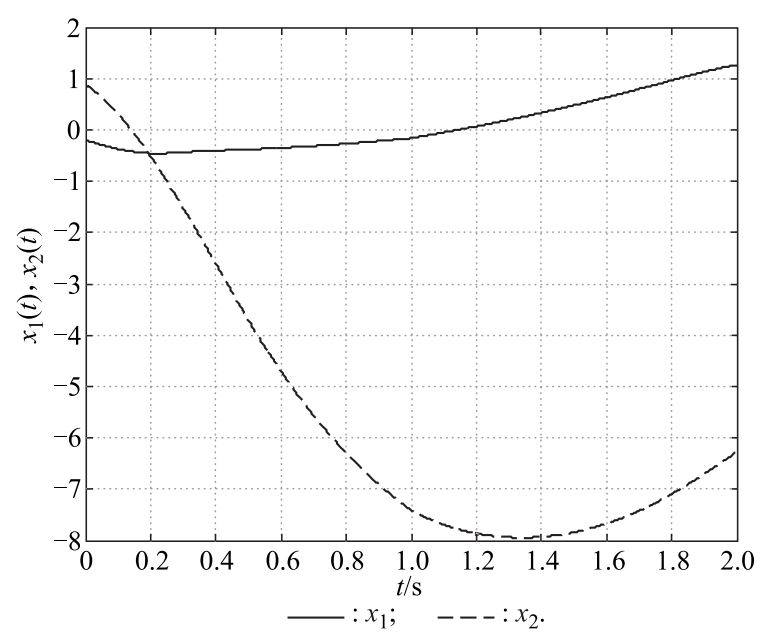

Fig. 5 Initial state trajectory of system (27) in Example 2

When the input is saturation, choose $\boldsymbol{G}=\operatorname{diag}(1,1)$, an AW compensator $\boldsymbol{E}_{c} \psi(\boldsymbol{K} \boldsymbol{x}(t))$ is added to system (27), so we can get system (4). Combined with (21), (22) and correlative data, the feasible results with the help of the LMI toolbox of Matlab are obtained:

$$
\begin{aligned}
\boldsymbol{P} & =\left[\begin{array}{ll}
4.0845 & 0.0390 \\
0.0390 & 0.9687
\end{array}\right], \\
\boldsymbol{Q} & =\left[\begin{array}{cc}
170.0805 & 5.6008 \\
5.6008 & 6.5276
\end{array}\right], \\
\boldsymbol{R} & =\left[\begin{array}{cc}
83.2961 & 1.1266 \\
1.1266 & 4.0304
\end{array}\right], \\
\boldsymbol{T} & =\left[\begin{array}{cc}
0.3016 & 0 \\
0 & 0.3016
\end{array}\right], \\
\boldsymbol{L} & =\left[\begin{array}{cc}
10.3724 & 2.8226 \\
-3.2476 & 15.4272
\end{array}\right], \\
\boldsymbol{J} & =\left[\begin{array}{cc}
13.4075 & -3.2457 \\
2.8272 & 18.1692
\end{array}\right],
\end{aligned}
$$

$$
\begin{gathered}
\boldsymbol{N}=\left[\begin{array}{cc}
-10.0914 & -2.8272 \\
3.2457 & -14.8532
\end{array}\right], \\
\boldsymbol{K}=\left[\begin{array}{cc}
42.4766 & 3.1391 \\
-12.6630 & 14.8180
\end{array}\right], \\
\boldsymbol{E}_{c}=\left[\begin{array}{cc}
4.0432 & -0.9788 \\
0.8526 & 5.4792
\end{array}\right] .
\end{gathered}
$$

The states of $x_{1}$ and $x_{2}$ of system (4) are shown in Fig. 6. The control locus diagrams of system (4) are shown in Fig. 7.

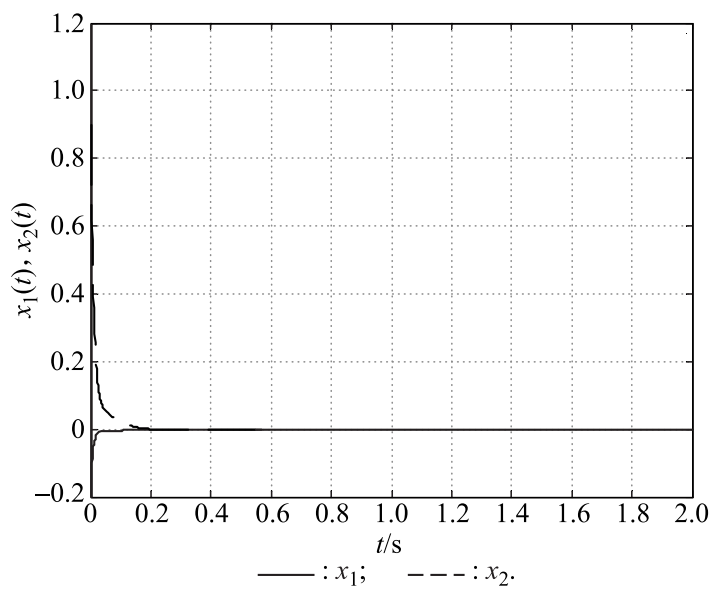

Fig. 6 State locus of system (4) with AW structure in Example 2

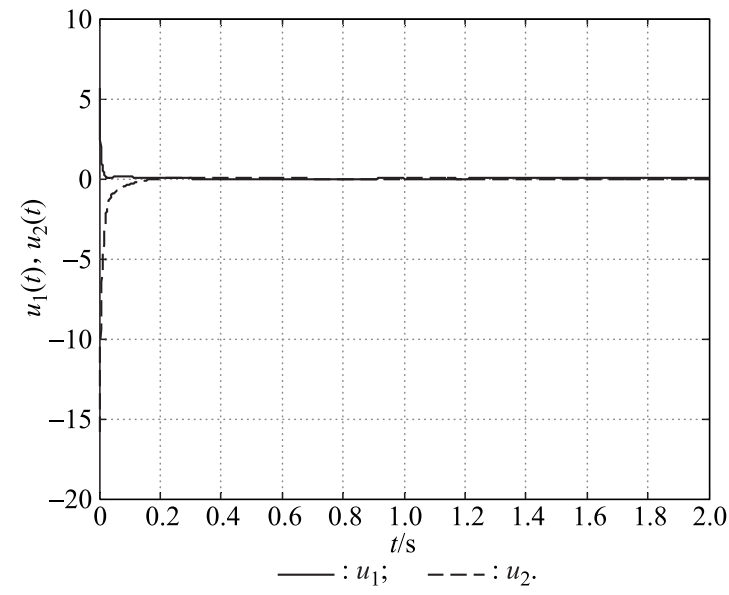

Fig. 7 Control locus of system (4) in Example 2

The same with Example 1, comparing Fig. 5 and Fig. 6, the open-loop system (27) is unstable at the setting initial state. By adding the designed AW structure, the closedloop system (4) becomes stable in a short time. The control locus of system (4) in Fig. 7 shows that $u_{1}(t)$ and $u_{2}(t)$ are big at the beginning. It is effective to make the state back to zero as soon as possible.

By solving (25), we can obtain another group of solution as follows:

$$
\boldsymbol{P}=\left[\begin{array}{cc}
0.0335 & -0.0014 \\
-0.0014 & 0.0141
\end{array}\right]
$$




$$
\begin{gathered}
\boldsymbol{Q}=\left[\begin{array}{ll}
1.8662 & 0.0781 \\
0.0781 & 0.0942
\end{array}\right] \\
\boldsymbol{R}=\left[\begin{array}{cc}
0.2500 & -0.0163 \\
-0.0163 & 0.0539
\end{array}\right] \\
\boldsymbol{T}=\left[\begin{array}{cc}
0.0093 & 0 \\
0 & 0.0093
\end{array}\right] \\
\boldsymbol{L}=\left[\begin{array}{cc}
1146.4 & 106.6137 \\
112.1377 & 1042.5
\end{array}\right] \\
\boldsymbol{J}=\left[\begin{array}{cc}
1250.9 & 112.0106 \\
106.4984 & 1145.3
\end{array}\right] \\
\boldsymbol{N}=\left[\begin{array}{cc}
-1143.3 & -106.4984 \\
-112.0106 & -1037.7
\end{array}\right] \\
\boldsymbol{K}=\left[\begin{array}{cc}
38.2195 & -0.0978 \\
2.2986 & 14.5271
\end{array}\right] \\
\boldsymbol{E}_{c}=\left[\begin{array}{cc}
11.6290 & 1.0413 \\
0.9901 & 10.6472
\end{array}\right], \quad \mu=0.0557 .
\end{gathered}
$$

The estimation of the domain of attraction of system (4) is shown in Fig. 8. In Fig. 8, one locus is the estimation of the domain of attraction before optimization (discrete), the other is the estimation of the domain of attraction after optimization (continuous). It is obvious that by solving (25), we obtain a larger estimate of the domain attraction.

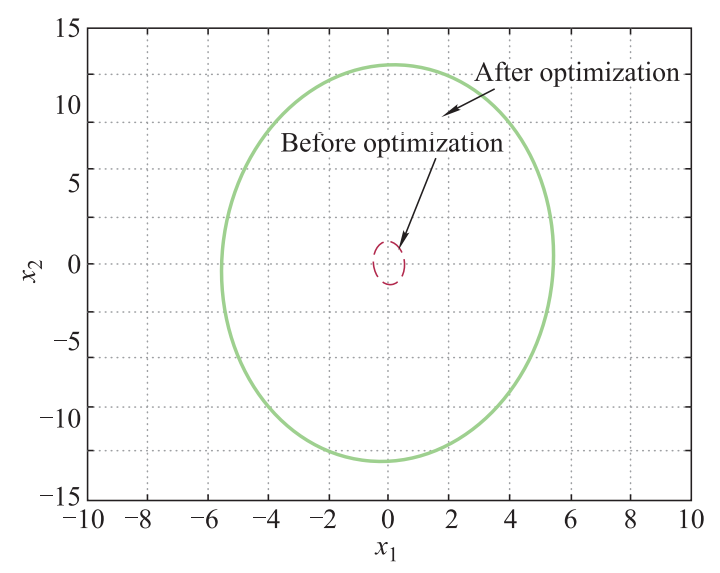

Fig. 8 Domain of attraction in Example 2

\section{Conclusions}

In this article, we deal with the stability problem for a class of distributed time-delayed CNNs with input saturation. Few papers discussed it before. Based on the Lyapunov theory, Schur complement principle and some lemmas, we propose an AW structure for CNNs with both constant time-delays and distributed time-delays. It is important to emphasize that for the structure of systems with $f(\boldsymbol{x}(t))$ and without $f(\boldsymbol{x}(t))(\boldsymbol{M} \neq 0$ and $\boldsymbol{M}=0)$, the stability condition has to be discussed separately. By solving a convex optimization problem, we get a larger domain of attraction. In the simulation section, the comparison between systems with and without considering input saturation fully testifies to the role and the superiority of the AW compensator in the stabilizing system.

\section{References}

[1] LI X M. Stability of cellular neural network. Changsha, China: Hunan University, 2002. (in Chinese)

[2] YU L F. Dynamical behavior of two classes of neural networks with time delays. Qingdao, China: Ocean University of China, 2008. (in Chinese)

[3] CHUA L O, YANG L. Cellular neural networks: theory and applications. IEEE Trans. on Circuits and Systems, 1988, 35(10): $1257-1290$.

[4] WEI L, WANG Z Y, YU Y M. Image restoration method based on neural network combining with optimization algorithm. Journal of Sichuan University (Natural Science Edition), 2011, 48(5): 1081 - 1086. (in Chinese)

[5] NOROUZI B, MIRZAKUCHAKI S. An image encryption algorithm based on DNA sequence operations and cellular neural network. Multimedia Tools and Applications, 2017, 76(11): $13681-13701$.

[6] JI N, XUN D Z, LIU F. A novel adaptive neural network constrained control for solid oxide fuel cells via dynamic antiwindup. Neurocomputing, 2016, 214: 134-142.

[7] JAMROZIK W. Cellular neural networks for welding arc thermograms segmentation. Infrared Physics \& Technology, 2014, 66: $18-28$.

[8] RATNAVELU K, KALPANA M, BALASUBRAMANIAM P, et al. Image encryption method based on chaotic fuzzy cellular neural networks. Signal Processing, 2017, 140: 87-96.

[9] MACHOT F A, ALI MMOSA A H, et al. Real-time raindrop detection based on cellular neural networks for ADAS. Journal of Real-Time Image Processing, 2016, 16(4): 931-943.

[10] ZHAI D H, XIA Y Q. Finite-time control of teleoperation systems with input saturation and varying time delay. IEEE Trans. on Systems, Man, and Cybernetics: Systems, 2017, 47(7): $1522-1534$

[11] BILOTTA E, PANTANO P, VENA S. Speeding up cellular neural network processing ability by embodying memristors. IEEE Trans. on Neural Networks and Learning Systems, 2017, 28(5): $1228-1232$.

[12] LAURET P, HEYMES F, APRIN L, et al. Atmospheric dispersion modeling using artificial neural network based cellular automata. Environmental Modelling \& Software, 2016, 85: 56-69.

[13] ZHANG Q, XU J. Global exponential stability of cellular neural networks. Journal of Electronics, 2000, 22(3): 434-438. (in Chinese)

[14] XI D M, HE H K, QIU J Q, et al. Delay-dependent global asymptotic stability criteria for a class of cellular neural networks. Journal of Hebei University of Science and Technology, 2008, 29(3): 188-193. (in Chinese)

[15] LIAO X F, LI X M , ZHOU S B. Stability analysis for delayed cellular neural networks based on linear matrix inequality approach. Chinese Journal of Computers, 2004, 27(3): 377 - 381. (in Chinese)

[16] ZHAO S Q. The global stability and synchronization of the cellular neural networks with delays. Tianjin, China: Tianjin Normal University, 2015. (in Chinese)

[17] XU C J. Global exponential convergence of fuzzy cellular neural networks with proportional delays and impulsive effects. 
Journal of Intelligent \& Fuzzy Systems, 2017, 33: 969-977.

[18] LIU B W. Finite-time stability of CNNs with neutral proportional delays and time-varying leakage delays. Mathematical Methods in the Applied Sciences, 2017, 40: 167-174.

[19] ZHOU L Q. Exponential stability of a class of cellular neural networks with unbounded delays. Chinese Journal of Engineering Mathematics, 2014, 31(4): 493-500. (in Chinese)

[20] ZHOU Q Y. Weighted pseudo anti-periodic solutions for cellular neural networks with mixed delays. Asian Journal of Control, 2017, 19(4): $1557-1563$.

[21] DU Y H, WEN W, ZHONG S M, et al. Complete delaydecomposing approach to exponential stability for uncertain cellular neural networks with discrete and distributed timevarying delays. International Journal of Control, Automation and Systems, 2016, 14(4): $1012-1020$.

[22] SONG X L, XIN X, HUANG W P. Exponential stability of delayed and impulsive cellular neural networks with partially Lipschitz continuous activation functions. Neural Networks, 2012, 29/30: 80-90.

[23] FANG S, JIANG M, FU W. Global exponential stability and periodicity of CNNs with time-varying discrete and distributed delays, advances in neural networks. Berlin: Springer, 2008.

[24] XIANG Z Y, ZHONG S M, LUO H. Global exponential stability for impulsive cellular neural networks with discrete and distributed time-varying delays. Proc. of the IEEE International Conference on Apperceiving Computing \& Intelligence Analysis, 2009: 373-376.

[25] ZHOU L, HU G. Global exponential periodicity and stability of cellular neural networks with variable and distributed delays. Applied Mathematics \& Computation, 2008, 195(2): $402-411$.

[26] CHAOUKI A, EL A A, JINDE C, et al. Global exponential convergence of neutral-type competitive neural networks with multi-proportional delays, distributed delays and time-varying delay in leakage delays. International Journal of Systems Science, 2018, 49(6): 1-13.

[27] GONG W, LIANG J, ZHANG C. Multi-stability of complexvalued neural networks with distributed delays. Neural Computing \& Applications, 2016, 28(1): 1-14.

[28] TARBOURIECH S, TURNER M. Anti-windup design: an overview of some recent advances and open problems. IET Control Theory and Applications, 2009, 3(1): 1-19.

[29] LI Q, SHEN B, LIU Y, et al. Event-triggered $\mathrm{H} \infty$ state estimation for discrete-time neural networks with mixed time delays and sensor saturations. Neural Computing \& Applications, 2017, 28(12): 3815-3825.

[30] ZENG H B, TEO K L, HE Y, et al. Sampled-data synchronization control for chaotic neural networks subject to actuator saturation. Neurocomputing, 2017, 185(10): 25-31.

[31] ZHOU S, CHEN M, ONG C J, et al. Adaptive neural network control of uncertain MIMO nonlinear systems with input saturation. Neural Computing \& Applications, 2016, 27(5): 1317 1325.

[32] GOMES DA SILVA JR J M, TARBOURIECH S. Anti-windup design with guaranteed regions of stability: an LMI-based approach. IEEE Trans. on Automatic Control, 2005, 50(1): 106111.

[33] TARBOURIECH S, GARCIA G, GOMES DA SILVA JR J M, et al. An overview of anti-windup techniques, in stability and stabilization of linear systems with saturating actuators. London: Springer-Verlag, 2011.

[34] ZHA M, HE H L. An overview of anti-windup design development. Journal of Nanjing University of information Science and Technology (Natural Science Edition), 2017, 9(4): 16-22. (in Chinese)

[35] MANZAR M N, BATTISTELLI G, SEDIGH A K. Input- constrained multi-model unfalsified switching control. Automatica, 2017, 83: 391-395.

[36] YAO X M, GUO L, WU L G, et al. Static anti-windup design for nonlinear Markovian jump systems with multiple disturbances. Information Science, 2017, 418/419: 169-183.

[37] FLESCH R C C, NORMEYRICO J E, FLESCH C A. A unified anti-windup strategy for SISO discrete dead-time compensators. Control Engineering Practice, 2017, 69: 50-60.

[38] TAHOUN A H. Anti-windup adaptive PID control design for a class of uncertain chaotic systems with input saturation. ISA Transactions, 2017, 66: 176-184.

[39] QI W H, PARK J H, CHENG J, et al. Anti-windup design for stochastic Markovian switching systems with mode-dependent time-varying delays and saturation nonlinearity. Nonlinear Analysis: Hybrid Systems, 2017, 26: $201-211$.

[40] GAYADEEN S, DUNCAN S R. Discrete-time anti-windup compensation for synchrotron electron beam controllers with rate constrained actuators. Automatica, 2016, 67: 224-232.

[41] LI J X, FANG Y M, SHI S L. Robust dynamic output-feedback control of hydraulic servo system with input saturation for rolling mill. Control and Decision, 2013, 28(2): 211 -216. (in Chinese)

[42] NGUYEN A T, DEQUIDT A, DAMBRINE M. Anti-windup based dynamic output feedback controller design with performance consideration for constrained Takagi-Sugeno systems. Engineering Applications of Artificial Intelligence, 2015, 40: $76-83$.

[43] KAO Y G, XIE J, WANG C H. An anti-windup design to robust $\mathrm{H} 1$ control for singular Markovian jump systems with actuator saturation and general unknown transition rates. Journal of the Franklin Institute, 2015, 352: 5708-5734.

[44] PATI A, NEGI R. An anti-windup control strategy to actuator saturating input voltage for active magnetic bearing system. The International Journal for Computation and Mathematics in Electrical and Electronic Engineering, 2016, 35(3): $1046-$ 1063.

[45] YAN L, HE H L, XIONG P. Static anti-windup scheme for state linearizable nonlinear control systems with saturating inputs. Proc. of the 33rd IEEE Chinese Control Conference, 2014: $1783-1787$

[46] HE H L, XU W W, JIANG M. Guaranteed cost anti-windup stabilization of discrete delayed cellular neural networks. Neural Process Letters, 2017, 46(1): 343-354.

[47] ZHA M, HE H L. One step anti-windup design for a class of time-delayed cellular neural networks. Proc. of the 2nd IEEE Advanced Information Management, Communicates, Electronic and Automation Control Conference, 2018: 1832 1837.

[48] TU J J, HE H L. Guaranteed cost synchronization of chaotic cellular neural networks with time-varying delay. Neural Computation, 2012, 24: $217-233$.

\section{Biographies}

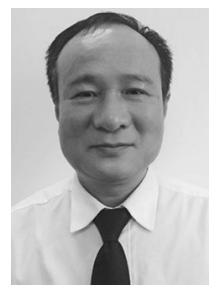

HE Hanlin was born in 1962. He received his B.S. degree in mathematics from Central China Normal University, Wuhan, China, in 1983. He received his M.S. degree in applied mathematics from Chongqing University, Chongqing, China, in 1989, and his Ph.D. degree in control science and engineering from Huazhong University of Science and Technology, Wuhan, China, in 2003. From 1990 to 1999 , he was a lecturer with Naval University of Engineering. From 2001 to 2005, he was an associate professor. Since 2006, he has been a professor with the Department of Basic Courses, Naval 
University of Engineering. His research interests include feedback control, fuzzy control, cellular neural networks control, anti-windup control, nonlinear systems, Lurie systems, chaos control and synchronization.

E-mail: hanlinhe62@aliyun.com

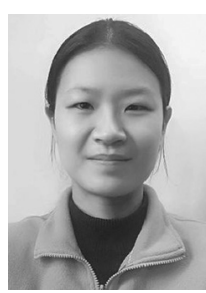

ZHA Miao was born in 1991. She received her B.S. degree in electronic information engineering from China University of Geosciences, Wuhan, China, in 2013 and M.S. degree in communication and information system from Naval University of Engineering, Wuhan, China, in 2015. She is currently pursuing her Ph.D. degree in control science and engineering at Naval University of Engineering, Wuhan, China. Her research interests include feedback control, anti-windup control, and nonlinear systems.

E-mail: zha30@qq.com

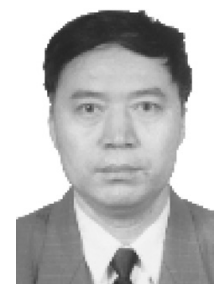

BIAN Shaofeng was born in 1961. He received his B.S. degree in geodesy and M.S. degree in astronomical geodesy in Institute of Surveying and Mapping, the PLA Information Engineering University, Zhengzhou, China, in 1982 and 1985, respectively and Ph.D. degree from Wuhan Technical University of Surveying and Mapping in 1992. He is a professor in the Department of Navigation Engineering, Naval University of Engineering. He was awarded an Alexander von Humboldt Research Fellowship in 1996. He got funded by the National Science Foundation for Distinguished Young Scholars, in 2001. His research interests include satellite navigation and geodesy.

E-mail: sfbian@sina.com 